\title{
Elaboración de un modelo que mejore los despachos nacionales dentro de una empresa de agroquímicos en Colombia
}

\author{
Carlos Alberto Miranda Abaúnza \\ Facultad de Ciencias Navales y Naúticas, Escuela Naval de Cadetes "Almirante Padilla”, Cartagena, Colombia. \\ kbtto@yahoo.com
}

Recibido: Septiembre 10, 2019.

Recibido en su versión corregida: Octubre 03, 2019.

Aceptación: Octubre 25, 2019.

Como citar: Miranda Abaúnza, C. A. (2019). Elaboración de un modelo que mejore los despachos nacionales dentro de una empresa de agroquímicos en Colombia. Revista Sextante, 20, pp. 22-32, 2019.

\section{Resumen}

Este artículo presenta el caso real de una empresa de agroquímicos domiciliada en Colombia, que permitió que fuera objeto de análisis el proceso de distribución a sus clientes, con el objetivo de encontrar una mejor forma de operar. El problema presentado es: ¿cómo puede hacerse de mejor manera algo que está en funcionamiento? Mediante el análisis de varias investigaciones y modelos se determina cómo se debe proceder con la modelación requerida. El resultado de este ejercicio es un trabajo donde los procesos logísticos vigentes son analizados a la luz de procedimientos de investigación, obteniendo no sólo un diagnóstico acertado, sino también mejoras plausibles, medibles y sostenibles en sus procesos logísticos.

Palabras claves: Administración logística, Diseño para la logística (DFL), Diagnóstico estratégico.

\section{Making of a model that improves national dispatch within an agrochemical company in Colombia}

\begin{abstract}
This paper presents the real case of an agrochemical company domiciled in Colombia that allowed the distribution process to its clients to be analyzed, in order to find a better way to operate. So the problem presented is, how can be done in a better way something in fact is already in operation. Through the analysis of several investigations and models, it is determined how to proceed with the required modeling and the result of this exercise is an academic work where the logistic processes in force are analyzed by research procedures with not only obtaining an accurate diagnosis but also believable, measurable and sustainable improvements in their logistics processes.
\end{abstract}

Keywords: Logistics management, Design for logistics (DFL), Strategic diagnosis. 


\section{Introducción}

Al momento de iniciar esta investigación la empresa de agroquímicos utilizada como referencia, cargaba sus vehículos para distribución utilizando el modelo de carga suelta, basándose en el conocimiento práctico adquirido por el personal responsable de ejecutar dichos cargues, por lo que la empresa no poseía información proveniente de análisis estadísticos ni de evaluación de operaciones. De igual forma desconocía las condiciones exactas y los recursos que tienen sus clientes a lo largo del territorio nacional, por lo que tampoco conocía el modelo más idóneo para el cargue, distribución y entrega de sus productos, tanto en la configuración de pallets en Colombia, como en la de carga suelta, lo que generaba demoras, ineficiencias y sobrecostos al interior de la cadena de suministro. Se requería un cambio en los procesos que lograra mejorar la operación, de manera segura y efectiva, beneficiando así, tanto la operación de distribución, como el almacenamiento.

La investigación permitió determinar cuál era el óptimo modelo de estandarización para el almacenamiento, prealistamiento, cargue y despacho y qué representaba la mayor efectividad en términos económicos, de tiempo, de servicio y de cubicaje para una empresa de agroquímicos ubicada en la ciudad de Cartagena, que distribuye alrededor de 7.500 toneladas al año, entre operaciones primarias y secundarias, a 12 clientes, con 23 puntos de entrega, en 20 ciudades. Para lograr el objetivo primero hubo que determinar la actualidad de dichos procesos, los recursos disponibles y la real capacidad operativa de la planta objeto de estudio, toda vez que al momento de iniciar la investigación estaba indeterminada.

\section{Metodología}

Para la investigación se utilizó la metodología del diseño para la logística, teniendo en cuenta las particularidades de la empresa. Dentro de la literatura que sustentó la teoría a aplicar, fue crítica la investigación de Mantilla (2009) para el diseño de red y las medidas de desempeño inherentes a las operaciones de distribución. En este sentido cobra vital importancia su investigación referente a modelos de desarrollo aplicados a la logística. Mantilla parte de la importancia capital que las actividades de apoyo tienen dentro del conjunto total de operaciones de una organización dedicada a la manufactura y transformación de bienes y señala cómo su optimización ha venido siendo un factor clave en el mejoramiento continuo de los procesos: "Varios estudios han demostrado que las actividades de manufactura conforman una tercera parte del tiempo productivo de la cadena de suministro y el resto del tiempo se emplea en procesamiento de órdenes, ingeniería, compras, instalación y esperas...Stalk and Hout plantean que la porción de tiempo en la que realmente se agrega valor está entre 0.05\%-5\% del tiempo de entrega. Debido a este desconocimiento del potencial de mejora en actividades diferentes a las productivas, se han generado a través de los años metodologías como JIT (Justo a tiempo), TPS (Sistema de producción Toyota), TQM (Administración total de la calidad), Lean manufacturing (Manufactura esbelta) y Six sigma (Seis sigmas) enfocadas básicamente en la manufactura, sin embargo, es necesario pensar cómo mejorar las otras dos terceras partes de la cadena" (Mantilla, 2009, p. 4)

Avanzando en su investigación, Mantilla (2009) propone un esquema de desarrollo basado en lo que define como un modelo de desarrollo estratégico basado en logística y al que llama Lean Six Sigma Logistics ó LSSL, utilizando como plataforma las metodologías de 'Lean Manufacturing' y 'Six Sigma'.

A la par de los avances y metodología desarrollados por Mantilla, la investigación también tuvo en cuenta diseños de operación más específicos para los problemas puntuales al interior de las gestiones logísticas de una organización como la empresa utilizada como objeto de la investigación. Para ello, esta investigación también se soportó en la obra de Bowersox, Closs \& Cooper (2007), quienes desarrollaron una metodología para lo que han llamado un "Diseño de la Logística y Planificación Operativa", pensado para utilizarse de la manera más universal posible, más allá de las obvias diferencias de enfoque entre una organización y otra,

El diseño de un sistema logístico debe evaluar diversas alternativas de servicio, características de costo y tecnologías operativas. Dichos análisis requieren un proceso estructurado y herramientas analíticas eficaces. Así como no existe un sistema logístico ideal que convenga a todas las empresas, 
el método para identificar y evaluar las estrategias logísticas alternas puede variar mucho. Sin embargo, existe un proceso general aplicable a casi todas las situaciones de diseño y análisis de la logística. Un proceso de planificación que se divide en tres fases: definición del problema $\mathrm{y}$ planificación, recopilación de datos y análisis, y recomendación e implementación. (Bowersox et al, 2007, p. 320)

Al final, toda la investigación debe soportarse en el correcto análisis de la cadena de suministro objeto de estudio, pues de la veraz obtención de la información primaria depende el éxito de esta. De ahí que la clave fue el conocimiento profundo y preciso de todo lo concerniente a la operación objetivo de la investigación, la realidad de sus recursos y procedimientos y la actualidad de su rango de acción.

A este punto, la investigación alcanzó el análisis del proceso logístico interno de la empresa, y en tal sentido, dicho análisis se convirtió en la piedra angular de todo el trabajo, pues es de la fiabilidad de los datos del diseño y de la planificación operativa donde se encuentra el núcleo del éxito de la investigación misma. Teniendo en cuenta la criticidad del análisis al proceso actual de la factoría, se plantea el uso de la metodología propuesta por Bowesox et al, de tal forma que el diseño del sistema logístico tendrá un asidero previo en el análisis del proceso logístico.
Así como no existe un sistema logístico ideal que convenga a todas las empresas, el método para identificar $\mathrm{y}$ evaluar las estrategias logísticas alternas puede variar mucho. Sin embargo, y tal como se mencionó anteriormente, existe un proceso general aplicable a casi todas las situaciones de diseño y análisis de la logística, el cual se divide en tres fases como se muestra en la Figura 1: definición del problema y planificación, recopilación de datos y análisis, y recomendación e implementación. El análisis del diseño de la cadena de suministro efectúa la evaluación estratégica de las alternativas implementadas como la contratación, la ubicación de las instalaciones, la ubicación de los almacenes, y las áreas del servicio en el mercado, las cuales son cada vez más importantes para optimizar los flujos para las cadenas de suministro globales.

\section{Resultados}

Los resultados encontrados con esta investigación se clasificaron en: caracterizaciones operativas, diagnóstico de operaciones logísticas (de acuerdo con su capacidad actual y estableciendo los KPIs de la operación), y el nuevo modelo logístico estandarizado, que fue efectivamente aplicado. Todos los resultados estuvieron de acuerdo con la aplicación de la metodología y marco teórico propuestos.
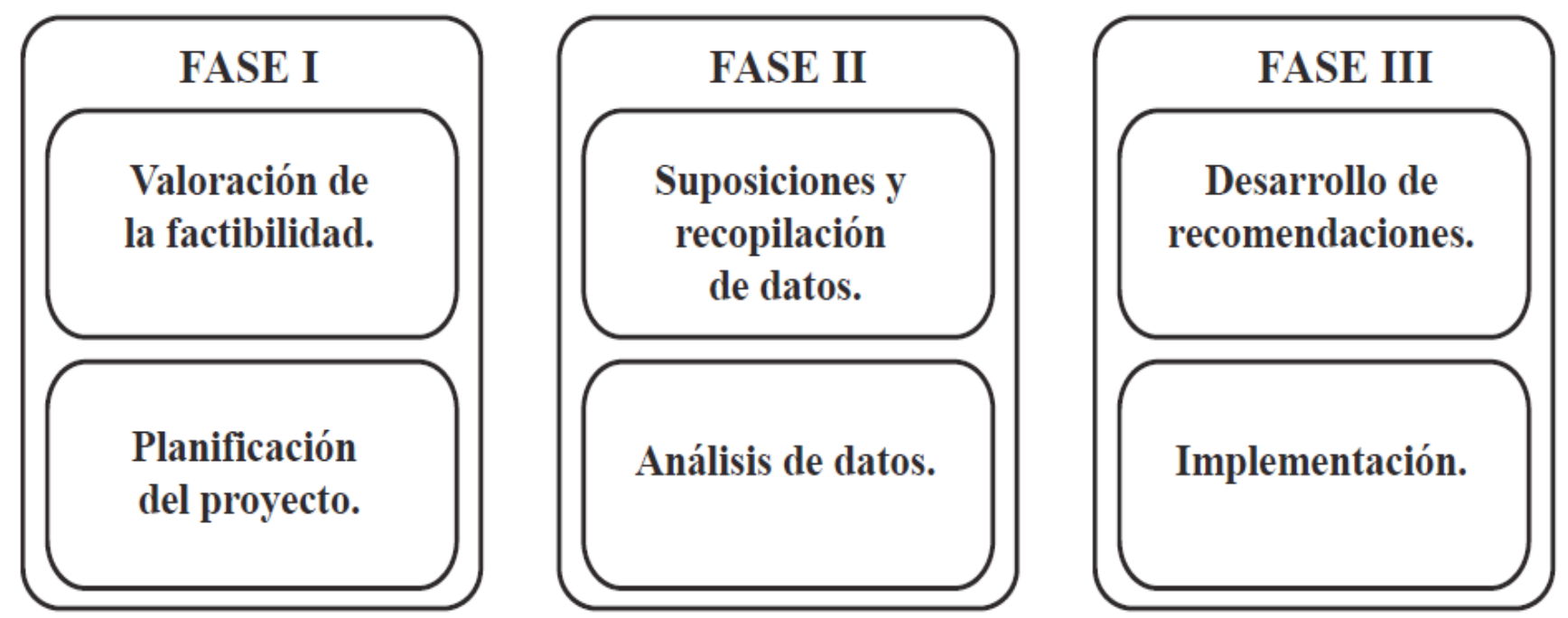

Figura 1. Metodología de la planificación.

Fuente: Bowesox et al, Administración y logística en la cadena de suministros, pág. 321. 


\section{Caracterizaciones operativas}

El desarrollo de las funciones logísticas al interior de la empresa implicó la segmentación en dos grandes grupos de operaciones: las de almacenamiento (que incluyen bodegaje, prealistamiento, cargue y despacho), y las de distribución (que son transporte, documentación y logística inversa). Teniendo en cuenta que el lead time de transporte y la logística inversa hacen parte de una operación de distribución tercerizada, las operaciones que requirieron especial énfasis a la hora de determinar su impacto en la mejora de los procesos fueron el prealistamiento, el cargue y el despacho. Así pues, con la caracterización de estos procesos, se inició la fase II del 'Diseño de logística y planificación operativa' planteado por Bowersox et $\mathrm{Al}$, consistente en la recopilación de datos y su posterior análisis.

\section{Prealistamiento}

El prealistamiento es el proceso mediante el cual las mercancías o productos a distribuir se preparan o prealistan para que, al momento del cargue, se encuentren consolidados y listos de tal forma que su cargue sea rápido y eficiente. El prealistamiento implica tener en cuenta las cantidades, referencias, clientes y destinos que se van a embarcar en un vehículo. Como ya se observó, la compañía tiene bastante atomizada su oferta de productos y segmenta más o menos de manera uniforme sus clientes y destinos. Eso hace que para un pedido típico haya varias referencias que deben enviarse, con diferentes cantidades y a distintos puntos de entrega, de tal suerte que es bastante probable que en un solo despacho - incluso los que son pocas toneladas para enviar en camiones pequeñospuedan significar el envío de diversas referencias en formato de carga suelta. Así pues, dentro de las operaciones logísticas de despacho de productos desde la planta de agroquímicos, el prealistamiento consiste en imprimir un listado de la mercancía a despachar donde conste las cantidades y destinos e informarles a los auxiliares de despachos para que con un montacarga se dediquen a separar los productos de sus estanterías y juntarlos en una zona para que al momento del cargue, este pueda suceder más rápidamente. Dentro de esta organización, dicho listado se emite desde el ERP que administra el inventario en sistema, y que para el caso de la empresa se trata del software SAP.
Con respecto a la antelación con la que se conocía el listado, en teoría estaba con un mínimo de 24 horas de antelación. Sin embargo, en la práctica el departamento de Servicio al cliente -que no está adscrito al área de Logística, sino a la de Ventas, la cual no está en la planta de Cartagena, sino en las oficinas de Bogotá- con frecuencia solicitaba cambios, adiciones o retiros de última hora, lo que complicaba la operación de prealistamiento y cargue.

\section{Cargue}

El cargue consiste en introducir al interior de los camiones los productos previamente seleccionados en el prealistamiento. Para tal efecto se traían las estibas antes separadas y con mano de obra se procedía con el cargue caja a caja, a excepción de los tambores de 208 litros, los cuales eran cargados con montacargas. Este proceso, sumado al hecho que un despacho típico tiene varias referencias y en un mismo camión hay varios destinatarios, hacía que en promedio un cargue tardara de 4 a 5 horas cuando se trataba de un tractocamión.

El hecho de que la mayoría de los cargues se hacen bajo el formato de carga suelta, implicaba que el cubicaje o acomodación de las cargas al interior del camión se hicieran basándose en la experiencia acumulada durante años por los auxiliares de cargue y de despacho. Así pues, al saber el tipo de producto, la cantidad a despachar, el número de destino y el tipo de camión a utilizar, los auxiliares definían el esquema de cargue dependiendo de cada caso. El envío de los productos en pallets -y que es de uso común por parte de otros competidores dentro del negocio de agroquímicos en Colombia- no había sido contemplado en el pasado.

\section{Despacho}

El despacho incluye tanto la documentación de envío como el seguimiento a la entrega. Dicha documentación consta de un manifiesto de carga o Carta de Porte (una hoja de seguridad (donde se consignan las propiedades químicas del producto a transportar y sus recomendaciones para el transporte y manejo de emergencias por filtración), y en ocasiones por solicitud de algunos clientes, de certificado de calidad emitido por la empresa. 
Respecto a los tiempos que la generación de los documentos tomaba, este variaba de acuerdo con la cantidad de referencias y el número de clientes, pero también por el número de pedidos que hacía un mismo cliente. A diferencia de otros manifiestos de carga similares emitidos en otras organizaciones, en esta compañía no existe un documento único que consolide todos los pedidos, sino que cada pedido genera su propia Carta de Porte. Así que, si incluso el cliente hace tres pedidos distintos de la misma referencia, al momento del despacho no se generará un solo documento por el total de una sola referencia, sino que habrá tres Cartas de Porte, cada una con las cantidades pedidas, aunque se trate del mismo producto. Teniendo en cuenta que es usual que en un mismo camión se envíen varios pedidos a distintos clientes, el número de documentos a imprimir y entregar al conductor crecía y los tiempos se alargaban, más si se estaban despachando varios vehículos al mismo tiempo.

\section{Diagnóstico de las operaciones logísticas}

El Análisis de los datos que hace parte de la metodología de Bowersox et al que se utiliza en la investigación, se planteó en formato de diagnóstico, utilizando la recopilación de datos acumulada hasta esta parte del estudio. Se pudo concluir que el desempeño alcanzado hasta el 2017 por el área de logística de la empresa atendió los requerimientos del área de ventas y soportó la operación de manera efectiva. Sin embargo, era posible mejorar sus índices, toda vez que muchos de sus procesos tenían evidentes oportunidades de mejora. El primer paso era establecer con claridad los factores claves para el éxito de sus operaciones logísticas. Por ello se determinó la actualidad de sus capacidades operativas y luego se presentaron los KPI que permitieron medir el ejercicio logístico de una manera objetiva, de tal forma que fuera posible comparar los índices de desempeño antes y después de la implementación de los ajustes propuestos por la investigación.

\section{Factores Clave}

Dentro de la operación logística de la compañía las variables, como la consecución de vehículos a tiempo, la utilización óptima del tipo de vehículo y su consecuente capacidad de carga, el aprovechamiento de las mismas rutas para varios clientes, el tiempo de carga y el prealistamiento resultaron tener una importancia distintiva y crítica.

Los procesos de distribución de los productos vendidos a los clientes iniciaban con el montaje de los pedidos por parte de los clientes. Si bien es cierto que la mayoría de los pedidos se piden con varios meses de antelación, la orden de despacho solía llegar una semana antes de la fecha de envío. Los clientes distribuidores se contactaban con el área de Servicio al Cliente para informarle qué requerimientos tenían respecto a reposición de sus inventarios o para casos de urgencia por parte de sus propios clientes. Salvo en estos últimos casos, los clientes distribuidores no suelen exigir un día específico para despachar. Los clientes de aplicación directa, en cambio, suelen ser muy exigentes a la hora de solicitar el envío de sus productos. Dado todo lo anterior, la decisión de fechas de envío estaba en manos del área de Servicio al Cliente y de Ventas, lo que hacía que los despachos no necesariamente tuvieran una previa evaluación de conveniencia logística que eventualmente optimizara los recursos sin afectar la promesa de entrega a los clientes. Así la solicitud de los vehículos a los proveedores de transporte y su posterior consecución y ubicación en la planta se veía influenciada de manera directa: desde Bogotá, Servicio al Cliente podía ordenar varios despachos durante la misma semana a un mismo cliente sin detenerse a ver la posibilidad de hacer un solo envío en un camión más grande. Esta decisión estratégica crítica es de índole logístico y rara vez le era consultada al equipo en Planta Cartagena.

En ese sentido, el tamaño y la frecuencia de los envíos dependían de las decisiones de Servicio al Cliente y/o Ventas. De hecho, el costo por tonelada transportada es una referencia que no se evalúa en dichas áreas y que obviamente es susceptible de mejorar si el peso por despacho aumenta. Respecto a la optimización de los espacios de cargue al interior de dichos camiones, no se encontraba establecida por medición alguna: todo dependía de la experticia que tuvieran los auxiliares de cargue y los auxiliares de despacho a la hora de cubicar. La utilización de alternativas que aprovechasen más eficientemente la capacidad de carga y transporte, tales como envíos paletizados, estaban muy limitadas por el desconocimiento que de ello tenían los clientes. 
Por último, fue evidente que el prealistamiento de los despachos y sus consecuentes tiempos de carga cambiarían sensiblemente si los tiempos de montaje de pedidos se estandarizaban. Lo realmente distintivo sería llegar a proceder con tal nivel de eficiencia en los cargues, que incrementarían las capacidades de despacho diarias sin tener necesidad de invertir en ampliaciones de muelle o incrementos de equipos y personal.

\section{Capacidad operativa actual}

Para el 2017 la empresa despachaba en promedio 2.7 vehículos por día. El tipo de camión más utilizado fue la tractomula. Aunque una prueba de toneladas promedio por vehículo despachado en 2017 arrojaba 18 tons/veh, la verdad es que es más cercano a la realidad diaria notar que los diferentes tipos de vehículos recorren diferentes tipos de rutas y lo que es aún más importante, diferentes optimizaciones de llenado.

De la Tabla 1, se observa que se hicieron un total de 718 despachos y dos quintas partes de estos correspondieron a envíos en vehículo tipo turbo. Aunque respecto a los camiones turbo hay que anotar que sólo transportaron el $11 \%$ del total de las toneladas distribuidas. La mayor cantidad de toneladas fueron enviadas en tractomulas $(51 \%$ del total), sin embargo, sólo fueron 143 de los 718 despachos efectuados en 2017 (20\% del total).

En total, para todos los tipos de vehículos en el 2017 los camiones llevaban en promedio un llenado del $84 \%$ del total de la capacidad, aunque con diferencia si se revisa que los vehículos turbo viajaron a sus destinos con una utilización promedio del $67 \%$, mientras que las tractomulas fueron despachadas con una utilización media del $88 \%$.

\section{KPIs de la operación}

Analizando las particularidades de los procesos logísticos de la compañía, se dedujeron los indicadores de gestión que le toman el pulso de la operación. Teniendo en cuenta que el objetivo de la presente investigación era diseñar un modelo que lograra mejorar las operaciones logísticas, los KPI debieron poder medir las toneladas distribuidas, la eficiencia de la capacidad instalada en los camiones, el número de despachos y el costo de transportar los productos a los clientes. Los indicadores de gestión clave para la operación investigada fueron entonces los siguientes:

- Toneladas Distribuidas (Tons): Número total de toneladas que el área de logística logró cargar, transportar y entregar durante un período. Sólo incluye las toneladas de distribución secundaria.

- Full Truck Load (FTL): Porcentaje de utilización de la capacidad de carga de los vehículos utilizados para la distribución. Mide la eficiencia en la distribución.

- Número de Despachos (Des): Cantidad total de despachos secundarios realizados por el área logística de la empresa. Se mide por cada envío, de tal manera que cada vehículo se cuenta como un despacho, indistintamente de las toneladas que se envíen en cada uno.

- Porcentaje de Utilización (\%Uso): Mide la relación entre las toneladas despachadas y el tipo de camión utilizado. Con este indicador se establece la tendencia general de envíos y las eventuales eficiencias a establecer dentro de los despachos.

Tabla 1. Número de despachos y porcentaje de uso 2017.

\begin{tabular}{cccccc}
\hline $\begin{array}{c}\text { Tipo de } \\
\text { vehículo }\end{array}$ & $\begin{array}{c}\text { Capacidad Max } \\
\text { de carga (Tons) }\end{array}$ & $\begin{array}{c}\text { Numero de } \\
\text { despachos }\end{array}$ & $\begin{array}{c}\text { Porcentaje de uso } \\
\text { sobre total de } \\
\text { despachos }\end{array}$ & $\begin{array}{c}\text { Porcentaje de uso } \\
\text { sobre total de } \\
\text { despachos }\end{array}$ & $\begin{array}{c}\text { Porcentaje de } \\
\text { toneladas sobre } \\
\text { total de despachos }\end{array}$ \\
\hline $\begin{array}{c}\text { Turbo } \\
\text { Sencillo }\end{array}$ & 4,5 & 288 & 869 & $40 \%$ & $11 \%$ \\
Dobletroque & 9 & 171 & 1185 & $24 \%$ & $15 \%$ \\
Minimula & 15 & 7 & 79 & $1 \%$ & $1 \%$ \\
Tractomula & 32 & 108 & 1738 & $15 \%$ & $22 \%$ \\
\hline & Total & $\mathbf{7 1 8}$ & 4029 & $20 \%$ & $51 \%$ \\
\hline
\end{tabular}

Fuente: Los autores. 
- Costo por Tonelada (\$/Ton): Mide lo que cuesta transportar una tonelada promedio dentro del territorio colombiano.

\section{Diseño de un modelo de estandarización}

El primer paso para iniciar un diseño de estandarización es aceptar que el mismo estará diseñado para las particularidades de la operación logística de la compañía, de tal forma que sus resultados se deben obtener con los datos suministrados por la misma y pensando en dicha organización como objeto final de estudio, por lo que no sería tan simple como extrapolar análisis similares y adaptarlos a la coyuntura. Se trata de un diseño hecho a la medida, con base en la administración logística y utilizando el método académico e investigativo soportado por los autores consultados para esta investigación, tal como Mantilla y Bowersox et al. Así las cosas, el diseño empezó por estandarizar el prealistamiento, procediendo luego con el cargue y concluyendo con lo propio para el despacho. Por último, establecieron los KPI para medición.

\section{Modelo de prealistamiento propuesto}

El modelo debió cambiar del anterior esquema reactivo a uno más proclive a incidir en las decisiones operacionales, de tal manera que pudiera influenciar positivamente en la gestión logística. En primer lugar, el área logística, como legítima conocedora de la operación, debía ser tenida en cuenta a la hora de tomar decisiones de cuánto, cuándo y cómo se les envían los pedidos a los clientes. Esto no quiere decir que debía o incluso pudiera reemplazar las decisiones estratégicas propias del área de ventas. Lo que se pretendía era poner al servicio de la organización sus conocimientos y experticia en la distribución, de tal manera que redundara en beneficios para la compañía.

La nueva procedimentación debía cambiar del anterior modelo, donde se podían hacer dos y hasta tres despachos en la misma semana, a uno donde se programasen despachos semanales a los clientes. Para ello, se requirió de la participación tanto del área de Ventas, como del visto bueno de las áreas Logísticas de los clientes. En el primer caso, Ventas no solo informó de manera anticipada la estrategia de ventas para el mes en cuestión (lo que obviamente no sólo debe incluir la meta de ventas o los planes especiales para cada cliente), sino que también expuso la tendencia de productos a despachar en el corto plazo. Esto permitió a la compañía conocer con la antelación suficiente los productos y las cantidades a despachar, por lo que tuvo a disposición la información requerida para programar con suficiencia los camiones. Sin embargo, el real impacto estuvo en que disminuyeron el número de despachos para las mismas toneladas. En otras palabras, la eficiencia medida en FTL y \$/Tons fue un resultado directo de este cambio: los pedidos se fueron acumulando y permitieron, no sólo la consolidación de carga -lo que implicó una reducción en el número de despachos y el aumento de los vehículos de mayor capacidad- sino que también tuvo la ventaja de que al cliente no le llegaran varios despachos en una semana, sino uno solo con todo lo que solicitó.

El proceso continuó con el prealistamiento a realizarse dentro de las 24 horas previas al despacho, de tal forma que los camiones programados estuvieran el menor tiempo posible en planta. Aunque esto parece demasiado evidente como para ser mencionado, es importante resaltar que este cambio en las operaciones implicó que las áreas de Servicio al Cliente y Ventas debían tener clara esta condición, pues era bastante común que éstos hicieran cambios de última hora en los productos y en sus cantidades, lo que obviamente retrasaba los despachos.

\section{Modelo de cargue propuesto}

Una vez los pedidos se encontraban prealistados desde el día anterior al cargue, el proceso se aceleró: el cargue no debió esperar a la búsqueda y separación de cantidades, sino que, por el contrario, procedió de manera eficiente, ya que el tener listos previamente los productos hizo más eficiente el proceso. Teniendo en cuenta que con el modelo anterior una tractomula se tardaba cerca de 7 horas en cargarse, la reducción en los tiempos de cargue trajo consigo optimizaciones tanto en el tiempo en general, como en la capacidad instalada, pues el área de logística dispuso de más tiempo para proceder con otras operaciones sin tener que recurrir a horas extra o en sobrecarga a los equipos.

De otra parte, el mayor cambio en el modelo de cargue estuvo en los despachos paletizados. Los 
productos se despachaban en formatos de carga suelta, por lo que las cajas se cargaban una a una al interior de los camiones. Como otra consecuencia del cambio de modelo de prealistamiento, se aumentaron las cantidades a despachar por cada envío y esto permitió contar con más cantidades de producto y tener cantidades mínimas paletizadas dentro de los despachos.

A fin de conocer el impacto que el cambio a paletización pudiera tener en los clientes, el autor de la presente investigación viajó a todos los puntos de entrega de todos los clientes, con el objeto de conocer de primera mano las condiciones y las capacidades de los clientes de la empresa. En las visitas se les consultó a los clientes si hacía diferencia recibir un solo despacho a la semana, si tenían equipos para el descargue de pallets, si tenían días específicos preferidos para el descargue, cómo almacenaban sus productos, y lo más importante, si para ellos hacía diferencia recibir sus pedidos en formato de pallet.

La respuesta de los clientes fue mayoritariamente a favor de la idea de recibir sus pedidos paletizados como se muestra en la Tabla 2.

\section{Modelo de despacho propuesto}

El nuevo modelo estándar de despachos incluye cambios sustantivos en la planeación y consecución de los vehículos programados para la distribución. Como se pudo establecer, los pedidos debían despacharse a más tardar en 48 horas luego del montaje de estos en el ERP. Esto implicaba que pudieran darse varios despachos dentro de las mismas rutas lógicas e incluso hacia los mismos clientes, perdiéndose así la posibilidad de consolidar carga. El modelo propuesto por la presente investigación implica estandarizar los despachos a frecuencias semanales para el caso de los clientes distribuidores, dándole días específicos a los despachos, dependiendo de su distancia y siempre teniendo en cuenta las rutas lógicas que permitan la optimización de las capacidades de carga de los camiones.

Teniendo en cuenta la planta de Cartagena como el origen de los despachos, existen dos tramos principales: las rutas que van hacia el suroccidente y que comprenden los destinos en:
Tabla 2. Disponibilidad de clientes para recibir en pallets.

\begin{tabular}{cccc} 
Ciudad & Nombre & Tons Año & $\begin{array}{c}\text { Acepta recibir } \\
\text { en Pallets }\end{array}$ \\
\hline Apartadó & Cliente $\mathrm{G}$ & 600 & $\mathrm{Si}$ \\
Apartadó & Cliente U & 150 & $\mathrm{No}$ \\
Bogotá & Cliente F & 75 & $\mathrm{Si}$ \\
Carepa & Cliente S & 188 & $\mathrm{Si}$ \\
Cartago & Cliente E & 450 & $\mathrm{Si}$ \\
Cereté & Cliente H & 1125 & $\mathrm{Si}$ \\
Cereté & Cliente I & 300 & $\mathrm{No}$ \\
Chigordogó & Cliente I & 19 & $\mathrm{Si}$ \\
Girón & Cliente I & 38 & $\mathrm{Si}$ \\
Granada & Cliente L & 98 & $\mathrm{Si}$ \\
Ibagué & Cliente I & 19 & $\mathrm{Si}$ \\
Neiva & Cliente C & 131 & $\mathrm{Si}$ \\
Pasto & Cliente D & 1050 & $\mathrm{Si}$ \\
Pereira & Cliente D & 600 & $\mathrm{Si}$ \\
Puerto Gaitán & Cliente H & 675 & $\mathrm{Si}$ \\
Sabaneta & Cliente I & 225 & $\mathrm{Si}$ \\
Santa Marta & Cliente B & 450 & $\mathrm{Si}$ \\
Santa Marta & Cliente U & 675 & $\mathrm{Si}$ \\
Tenjo & Cliente A & 75 & $\mathrm{Si}$ \\
Tunja & Cliente A & 19 & $\mathrm{Si}$ \\
Villavicencio & Cliente L & 225 & $\mathrm{Si}$ \\
Yopall & Cliente L & 300 & $\mathrm{Si}$ \\
Yumbo & Cliente D & 19 & $\mathrm{Si}$ \\
\hline
\end{tabular}

Fuente: Los autores.

Apartadó, Carepa, Chigorodó, Cereté, Sabaneta, Pereira, Cartago, Yumbo y Pasto; y las rutas que van hacia el suroriente, que comprenden los destinos a: Girón, Tunja, Tenjo, Bogotá, Villavicencio, Yopal, Granada, Puerto Gaitán, Neiva e Ibagué. El tramo suroccidental tiene dos rutas lógicas: aquellas que van al Urabá (Apartadó, Carepa y Chigorodó) y las que van hacia por todo el occidente colombiano hacia el sur y que comprende todas las demás ciudades. Ambas rutas pasan por Cereté, por lo que este destino servirá para adicionarle carga a las otras dos rutas.

El tramo suroriental tiene en común el trayecto hacia el centro del país por lo que los despachos a Girón, Tenjo, Tunja y Bogotá sirven a la hora de buscar consolidación de carga. A partir de ahí las rutas lógicas se dividen entre las que van hacia el oriente (Villavicencio, Yopal, Granada y Puerto Gaitán) y las que van hacia el Tolima Grande (Ibagué y Neiva).

Este nuevo modelo de planeación y ruteo debe entenderse como una guía para despachos que no va en menoscabo con el objetivo principal que es el de mejorar los procesos ya existentes. De esta forma el diseño de la ruta lógica óptima posible es prioritario, 
dándosele prelación al vehículo más grande para las distancias mayores.

El segundo aspecto positivo a tener en cuenta dentro del nuevo modelo de despacho va en la antelación con la que se les pide a los proveedores de transporte el vehículo. Dado que los tres proveedores (Amarillo, Azul y Rojo) tienen en las tractomulas y minimulas su fuerte principal y que el costo por tonelada se optimiza a medida en que el FTL de los vehículos más grandes tiende al 100\%, los problemas de consecución de vehículos se reducirían sustancialmente, pues la programación de los camiones pasaría de un día para otro a una semana de antelación.

Indicadores de gestión del nuevo modelo Aplicado

De acuerdo con lo planteado recientemente en los nuevos modelos de prealistamiento, cargue y despacho, y continuando el modelo de Diseño propuesto por Bowersox et $\mathrm{Al}$ en su Fase III, mediante los KPI se procedieron a medir las toneladas distribuidas, la eficiencia de la capacidad instalada en los camiones, el número de despachos, el costo de transportar los productos a los clientes y su respectivo comparativo con el modelo anterior, a fin de conocer el resultado del ejercicio de mejora objeto de este trabajo.

\section{A) KPI toneladas despachadas}

La Empresa de Agroquímicos objeto de la presente investigación tuvo un descenso del $5 \%$ en el número de toneladas transportadas en 2018 frente a las que se presentaron en 2017.

La reducción de las toneladas representó un reto para el modelo propuesto, pues la implementación de este trataba, entre otras cosas, de aumentar las toneladas por despacho, por lo que una reducción de 400 toneladas en comparación con el año anterior reducía el margen de maniobra y hacía aún más difícil la consolidación de pedidos $\mathrm{y}$, por consiguiente, de la carga, por lo que conocer el total de toneladas transportadas terminó teniendo gran trascendencia.

\section{B) KPI Full truck load}

La consolidación de carga que implicó el uso del nuevo modelo trajo los resultados esperados dentro de la optimización de la capacidad de carga de los vehículos. Como se dijo anteriormente, para el 2017 el promedio de llenado de los camiones estuvo en un $84 \%$. Para el 2018, en pleno uso del modelo nuevo, el FTL pasó a un 95\%, mejorando en todos los tipos de vehículos, pero especialmente en los que mayor impacto tienen sobre los costos, como son las tractomulas y las minimulas (ver Tabla 3).

En términos generales, la utilización de las capacidades de carga fue mejor respecto al año inmediatamente anterior y si se tiene en cuenta la reducción en el total de toneladas año, se puede reconocer la mejora en la optimización del recurso.

Como nota adicional, cabe resaltar que el incremento en el FTL para 2018 fue directamente proporcional al tipo de vehículo con mayor carga, lo que impactó directamente en el número de despachos realizados.

\section{C) KPI Número de despachos y porcentaje de uso}

Como consecuencia directa de la aplicación del nuevo modelo, y en concordancia con los indicadores de FTL y toneladas despachadas, el número de despachos también se vio afectado. De un total de 718 despachos realizados en 2017 (ver Tabla 1), se pasó a 363 despachos en 2018 (ver Tabla 4). Y más importante aún es que la utilización de los vehículos más pequeños se redujo en beneficio de los camiones con mayor capacidad.

Aunque los vehículos tipo turbo siguieron siendo una parte importante del uso general de camiones (64 despachos para un $18 \%$ del total), sufrieron una

Tabla 3. Full truck load 2017-2018.

\begin{tabular}{lll}
\hline \multirow{2}{*}{ Tipo de vehículo } & \multicolumn{2}{c}{ FLT } \\
\cline { 2 - 3 } & 2017 & 2018 \\
\hline Turbo & $67 \%$ & $78 \%$ \\
Sencillo & $77 \%$ & $81 \%$ \\
Dobletroque & $78 \%$ & $83 \%$ \\
Minimula & $89 \%$ & $92 \%$ \\
Tractomula & $88 \%$ & $97 \%$ \\
\hline \multicolumn{1}{c}{ Total } & $\mathbf{8 4 \%}$ & $\mathbf{9 5 \%}$ \\
\hline
\end{tabular}

Fuente: Los autores. 
Tabla 4. Número de despachos y porcentaje de uso 2018.

\begin{tabular}{cccccc}
\hline Tipo de vehículo & $\begin{array}{c}\text { Capacidad Max } \\
\text { de carga (Tons) }\end{array}$ & $\begin{array}{c}\text { Número de } \\
\text { despachos }\end{array}$ & $\begin{array}{c}\text { Toneladas } \\
\text { Movilizadas }\end{array}$ & $\begin{array}{c}\text { Porcentaje de uso } \\
\text { sobre el total de } \\
\text { despachos }\end{array}$ & $\begin{array}{c}\text { Porcentaje } \\
\text { Toneladas sobre } \\
\text { total despachos }\end{array}$ \\
\hline Turbo & 4.5 & 64 & 225 & $18 \%$ & $3 \%$ \\
Sencillo & 9 & 51 & 375 & $14 \%$ & $5 \%$ \\
Dobletroque & 15 & 6 & 75 & $2 \%$ & $1 \%$ \\
Minimula & 18 & 45 & 750 & $12 \%$ & $10 \%$ \\
Tractomula & 32 & 196 & 6075 & $54 \%$ & $81 \%$ \\
\hline & Total & 363 & 7500 & $100 \%$ & $100 \%$ \\
\hline
\end{tabular}

Fuente: Los autores.

Tabla 5. Costo por tonelada transportada 2017-2018.

\begin{tabular}{crrrr}
\hline Tipo de Vehículo & \multicolumn{4}{c}{$\begin{array}{c}\text { Costo por tonelada } \\
\text { (US\$) }\end{array}$} \\
\hline & \multicolumn{2}{c}{2017} & \multicolumn{2}{c}{2018} \\
\hline Turbo & $\$$ & 370 & $\$$ & 640 \\
Sencillo & $\$$ & 189 & $\$$ & 541 \\
Dobletroque & $\$$ & 110 & $\$$ & 110 \\
Minimula & $\$$ & 110 & $\$$ & 100 \\
Tractomula & $\$$ & 70 & $\$$ & 65 \\
\hline Total & $\$$ & $\mathbf{1 3 0}$ & $\mathbf{\$}$ & $\mathbf{1 1 0}$ \\
\hline
\end{tabular}

Fuente: Los autores.

reducción considerable si se tiene en cuenta que para el 2017 se realizaron 288 despachos en turbo (un $40 \%$ del total). Las tractomulas, en cambio, pasaron de 143 (20\% del total) a 196 despachos, llegando al 54\% del total de despachos.

$\mathrm{Si}$ adicional a lo anterior se observa que los vehículos turbo sólo transportaron el 3\% del total de toneladas, se entiende que el modelo implicó un cambio en la utilización de los vehículos haciendo que las tractomulas (el camión más eficiente para grandes distancias y costo por tonelada) transportaran para 2018, 6.075 de las 7.500 toneladas enviadas a los clientes. Todo esto redundó en el aumento de las toneladas por vehículo (pues se pasó de 11 a 21 toneladas promedio por despacho) y por ende en una reducción de los costos de distribución.

\section{D) KPI Costo por tonelada transportada}

El costo promedio por transportar una tonelada desde Cartagena hacia los distintos puntos de entrega de los clientes pasó de US\$130 en 2017 a US\$110 en 2018 (ver Tabla 5). Esta disminución en los costos totales de distribución se dio a pesar del aumento de los costos por tonelada transportada en turbos y sencillos, pues como se vio en los indicadores anteriores, el incremento en el uso de tractomulas y minimulas permitió que la reducción en los costos (así fueran proporcionalmente menores) impactara sustantiva y positivamente en los costos.

\section{Conclusiones}

En resumen, mediante la aplicación del nuevo modelo propuesto la empresa tuvo una reducción cercana al $15 \%$ sobre sus costos de transporte secundarios por tonelada, valor que se dio aún si tenemos en cuenta la reducción en las toneladas a transportar de un año a otro. Las reformas que se estructuraron en los procesos de Prealistamiento, Cargue y Despachos fueron incorporadas a la procedimentación estándar y se implementaron las mediciones de operación a través de los KPI sugeridos.

\section{Referencias}

Aristizábal, E., Gómez, J., y Londoño, G. (2008) Mapa de riesgo químico y por transporte de sustancias peligrosas en el Valle de Aburrá. Revista Universidad EAFIT, 44(150), 64-76.

Ballesteros, P., Ballesteros, D. y Bernal, M. (2006) Una forma práctica para hacer planeación estratégica logística, Scientia Et Technica, 12(30), Universidad Tecnológica de Pereira, 279-284.

Bowersox, D., Closs, D. y Cooper, B. (2007) Administración y logística en la cadena de suministros, México: McGraw-Hill Interamericana. 
Breval, S., Follmann, N., Pinheiro, O. y Rodríguez, C., (2017) Una nueva definición de la logística interna y forma de evaluar la misma, Revista Ingeniare, 25(2), Universidad de Tarapacá, 265.

Cippoletta, G., Pérez, G., y Sánchez R. (2010) Políticas integradas de infraestructura, transporte $y$ logística: experiencias internacionales y propuestas iniciales, serie Recursos Naturales e Infraestructura-CEPAL, Santiago de Chile: Naciones Unidas.

Mantilla, O. (2009) Lean Six Sigma Logistics: Modelo de Desarrollo-Edición Única, (Tesis de Maestría), Facultad de ingeniería, Instituto Tecnológico de Monterrey, Monterrey.

Mora, L. (2014) Logística del transporte $y$ distribución de carga, Bogotá ECOE Ediciones. 